\title{
The joint meeting of AAPS, JSPS, IPEG and WOFAPS
}

\author{
Tomoaki Taguchi ${ }^{1} \cdot$ Hiroaki Kitagawa ${ }^{2}$
}

Accepted: 3 January 2017 / Published online: 20 January 2017

(C) Springer-Verlag Berlin Heidelberg 2017

The joint meeting of the 24th Asian Association of Pediatric Surgeons (AAPS), 53rd Japanese Society of Pediatric Surgeons (JSPS), 25th International Pediatric Endosurgical Group (IPEG) and the World Federation of Associations of Pediatric Surgeons (WOFAPS) was held in Fukuoka, Japan, during 24-26th May 2016. This was a unique occasion because it is the first time ever that the four major pediatric surgery associations met together. The main theme of the meeting was "Harmony" representing Communication, Collaboration and Cooperation. The Organizing and Programme Committees made every effort to ensure that the anniversary meeting was International and educational for young Asian pediatric surgeons.

The meeting was very well attended with the total number of 1155 participants, including a large number of overseas guests from many countries. It was a very successful meeting with 832 papers presented relating to clinical pediatric surgery and pediatric surgery research.

There were nine International oral sessions that included Minimally Invasive Surgery, Pediatric Airway Disease, Neonatal and Fetal Surgery, Short Gut Syndrome, Pediatric Tumors, Trauma and Hepatobiliary disease with 3-6 Keynote lectures in each session. Suruga Lecture was delivered by Professor Takeshi Miyano from Juntendo Medical University in Tokyo. A total of 42 Keynote lectures were given by experts in their respective fields. Fourteen review articles were selected for publication in this special issue of PSI.

We, the guest editors of this special issue of PSI, are honored to share the contents of the highly successful meeting in Fukuoka with all the pediatric surgeons around the world.

Tomoaki Taguchi

taguchi@pedsurg.med.kyushu-u.ac.jp

Hiroaki Kitagawa

h2kita@marianna-u.ac.jp

1 Department of Pediatric Surgery, Graduate School of Medical Sciences, Kyushu University, Fukuoka 812-8582, Japan

2 Division of Pediatric Surgery, St. Marianna University School of Medicine, Kawasaki, Japan 